\title{
Approximate Solution of LR Fuzzy Matrix System
}

\author{
Dequan Shang ${ }^{1}$, Xingmin $\mathrm{Wei}^{1}$ and Xiaobin Guo ${ }^{2, *}$ \\ ${ }^{1}$ College of Public Health, Gansu University of Traditional Chinese Medicine, Lanzhou 730000, China \\ ${ }^{2}$ College of Mathematics and Statistics, Northwest Normal University, Lanzhou 730070, China \\ ${ }^{*}$ Corresponding author
}

\begin{abstract}
In this paper we proposed a general model for solving the fuzzy matrix equation $A X=B$ in which $A$ is a crisp matrix and $B$ is an arbitrary LR fuzzy numbers matrix. The original fuzzy equation is converted to a system of linear matrix equations by the embedding approach. The fuzzy solution is derived from solving model and a sufficient condition for the existence of strong LR fuzzy solution is analyzed. Two illustrating examples are given to show the effectiveness of the proposed method.
\end{abstract}

Keywords-LR fuzzy numbers; matrix analysis; fuzzy matrix equations; fuzzy approximate solutions

\section{INTRODUCTION}

To this day, there has a great enormous investigation in the study of fuzzy mathematics. From 1976 to 1991, some scholars studied fuzzy mathematics theory and obtained a series of results. Zadeh [1], Dubois et al.[2] and Nahmias [3] firstly introduced the concept of fuzzy numbers and arithmetic operations. Meanwhile, Puri and Ralescu [4], Goetschell et al.[5] and Wu Congxin et al.[6-7] investigated the structure of fuzzy number spaces. The uncertainty of the parameters is involved in the process of actual mathematical modeling, which is often represented by fuzzy numbers. Now the theory and computing method of fuzzy linear systems are still playing an important role to the fuzzy mathematics and its applications.

In 1998, Friedman et al.[8] proposed a method that they can transform $n \times n$ fuzzy linear systems into $2 n \times 2 n$ crisp function linear equation $S X(r)=Y(r)$ by using fuzzy set decomposition theorem and embedding method. Later, S. Abbasbandy et al.[9], T. Allahviranloo et al.[10-11], B. Zheng et al.[12] studied some specific fuzzy linear systems such as dual fuzzy linear systems, general fuzzy linear systems, dual full fuzzy linear systems and general dual fuzzy linear systems. In recent years, T. Allahviranloo, N. Babbar et al.[13-14] proposed many new approaches and theories of fuzzy linear systems. In 2009, Allahviranloo et al. [15] firstly discussed the fuzzy linear matrix equations (FLME) with form of $A \tilde{X} B=\tilde{C}$ by the Kronecker product of the matrices. In 2011, Gong and Guo[16] considered a class of fuzzy matrix equations $A \tilde{X}=\widetilde{B}$ and obtained its fuzzy least squares solutions by the embedding approach. In 2012, M. Otadi et al.[17] considered a kind of fully fuzzy matrix equation $\tilde{X} \otimes \tilde{A}=\widetilde{B}$. Recently, Guo et al.[18-19] proposed a computing method for complex fuzzy linear systems $C \tilde{z}=\tilde{w}$ and investigated fuzzy matrix equation with the form $\tilde{X} A=\widetilde{B}$ based on angular fuzzy numbers.
In this paper, we consider a class of LR fuzzy matrix equation $A \widetilde{X}=\widetilde{B}$ by a complete matrix method.

\section{PRELIMINARIES}

Definition 2.1 $A$ fuzzy number $\tilde{M}$ is said to be a LR fuzzy number if

$$
\mu_{\tilde{M}}(x)=\left\{\begin{array}{l}
L\left(\frac{m-x}{\alpha}\right), x \leq m, \alpha>0, \\
R\left(\frac{x-m}{\beta}\right), x \geq m, \beta>0,
\end{array}\right.
$$

where $m, \alpha$ and $\beta$ are called the mean value, left and right spreads of $\tilde{M}$, respectively. The function $L($.), which is called left shape function satisfies:

(1) $L(x)=L(-x)$,

(2) $L(0)=1$ and $L(1)=0$,

(3) $L(x)$ is a non increasing on $[0,+\infty)$.

The definition of a right shape function $R($.$) is similar to$ that of $L($.$) .$

Definition 2.2 For arbitrary LR fuzzy numbers $\tilde{M}=(m, \alpha, \beta)_{L R}$ and $\tilde{N}=(n, \gamma, \delta)_{L R}$, we have

(1) Addition

$$
\begin{aligned}
\tilde{M}+\tilde{N} & =(m, \alpha, \beta)_{L R}+(n, \gamma, \delta)_{L R} \\
& =(m+n, \alpha+\gamma, \beta+\delta)_{L R} .
\end{aligned}
$$

(2) Subtraction

$$
\begin{aligned}
\tilde{M}-\tilde{N} & =(m, \alpha, \beta)_{L R}-(n, \gamma, \delta)_{L R} \\
& =(m+n, \alpha+\gamma, \beta+\delta)_{L R}
\end{aligned}
$$

(3) Scalar multiplication

$$
\begin{aligned}
\lambda \tilde{M} & =\lambda(m, \alpha, \beta)_{L R} \\
& \cong\left\{\begin{array}{l}
(\lambda m, \lambda \alpha, \lambda \beta), \lambda \geq 0, \\
(\lambda m,-\lambda \beta,-\lambda \alpha)_{R L}, \lambda<0 .
\end{array}\right.
\end{aligned}
$$


Definition 2.3 A matrix $\tilde{A}=\left(\tilde{a}_{i j}\right)$ is called a LR fuzzy matrix, if each element $\tilde{a}_{i j}$ of $\tilde{A}$ is a LR fuzzy number.

Definition 2.4 The matrix system

$$
\begin{gathered}
\left(\begin{array}{cccc}
a_{11} & a_{12} & \cdots & a_{1 n} \\
a_{21} & a_{22} & \cdots & a_{2 n} \\
\cdots & \cdots & \cdots & \cdots \\
a_{m 1} & a_{n 2} & \cdots & a_{m n}
\end{array}\right)\left(\begin{array}{cccc}
\tilde{x}_{11} & \tilde{x}_{12} & \cdots & \tilde{x}_{1 p} \\
\tilde{x}_{21} & \tilde{x}_{22} & \cdots & \tilde{x}_{2 p} \\
\cdots & \cdots & \cdots \\
\tilde{x}_{n 1} & \tilde{x}_{n 2} & \cdots & \tilde{x}_{n p}
\end{array}\right) \\
\\
=\left(\begin{array}{cccc}
\tilde{b}_{11} & \tilde{b}_{12} & \cdots & \tilde{b}_{1 p} \\
\tilde{b}_{21} & \tilde{b}_{22} & \cdots & \tilde{b}_{2 p} \\
\cdots & \cdots & \cdots \\
\tilde{b}_{m 1} & \tilde{b}_{m 2} & \cdots & \tilde{b}_{m p}
\end{array}\right)
\end{gathered}
$$

where $a_{i j}, 1 \leq i \leq m, 1 \leq j \leq n$ are crisp numbers and $\tilde{b}_{i j}$, $1 \leq i \leq m, 1 \leq j \leq p$ are LR fuzzy numbers, is called a LR fuzzy matrix equations (LRFLME).

Using matrix notation, we have

$$
A \tilde{X}=\widetilde{B}
$$

A fuzzy numbers matrix $\tilde{X}$ is called a solution of the fuzzy matrix equation (5) if $\tilde{X}$ satisfies $A \tilde{X}=\tilde{B}$.

\section{SOLVING FUZZY MATRIX EQUATION}

Theorem 3.1 The fuzzy linear system $A \tilde{X}=\widetilde{B}$ can be extended into the following system of linear matrix equations

$$
\left\{\begin{array}{l}
\left(A^{+}+A^{-}\right) X=B \\
\left(\begin{array}{cc}
A^{+} & -A^{-} \\
-A^{-} & A^{+}
\end{array}\right)\left(\begin{array}{l}
X^{l} \\
X^{r}
\end{array}\right)=\left(\begin{array}{l}
B^{l} \\
B^{r}
\end{array}\right)
\end{array}\right.
$$

where $\tilde{X}=\left(X, X^{l}, X^{r}\right)$.

Proof. We denote the right fuzzy matrix $\widetilde{B}$ with $\widetilde{B}=\left(B, B^{l}, B^{r}\right)=\left(b_{i j}, b_{i j}^{l}, b^{r}{ }_{i j}\right)_{m \times p}$ and the unknown fuzzy matrix $\tilde{X}$ by

$$
\tilde{X}=\left(X, X^{l}, X^{r}\right)=\left(x_{i j}, x_{i j}^{l}, x^{r}{ }_{i j}\right)_{n \times p} .
$$

We also suppose $A=A^{+}+A^{-}$in which the elements $a^{+}{ }_{i j}$ of matrix $A^{+}$and of $a^{-}$ij matrix $A^{-}$are determind by the following way:

if $a^{+}{ }_{i j} \geq 0, a^{+}{ }_{i j}=a_{i j}$ else $a^{+}{ }_{i j}=0,1 \leq i \leq m, 1 \leq j \leq n$; if $a^{+}{ }_{i j}<0, a^{-}{ }_{i j}=a_{i j}$ else $a^{-}{ }_{i j}=0,1 \leq i \leq m, 1 \leq j \leq n$.

For fuzzy matrix equation $\tilde{X} A=\widetilde{B}$, we can express it as

$$
\left(A^{+}+A^{-}\right)\left(X, X^{l}, X^{r}\right)=\left(B, B^{l}, B^{r}\right)
$$

Since

$$
k \tilde{x}_{i j}=\left\{\begin{array}{ll}
\left(k x_{i j}, k x_{i j}^{l}, k x^{r}{ }_{i j}\right), & k \geq 0, \\
\left(k x_{i j},-k x_{i j}^{r},-k x_{i j}^{l}\right), & k<0
\end{array},\right.
$$

we have

$$
A \tilde{X}= \begin{cases}\left(A X, A X^{l}, A X^{r}\right), & A \geq 0, \\ \left(A X,-A X^{r},-A X^{l}\right), & A<0\end{cases}
$$

So the Eq.(7) can be rewritten as

$$
\begin{aligned}
& A^{+}\left(X, X^{l}, X^{r}\right)+A^{-}\left(X, X^{l}, X^{r}\right)=\left(A^{+} X, A^{+} X^{l}, A^{+} X^{r}\right)+ \\
& \left(A^{-} X,-A^{-} X^{l},-A^{-} X^{r}\right) \\
& \left(A^{+} X+A^{-} X, A^{+} X^{l}-A^{-} X^{r}, A^{+} X^{r}-A^{-} X^{l}\right)=\left(B, B^{l}, B^{r}\right) .
\end{aligned}
$$

Thus we get

$$
\left\{\begin{array}{l}
A^{+} X+A^{-} X=B, \\
A^{+} X^{l}-A^{-} X^{r}=B^{l}, \\
A^{+} X^{r}-A^{-} X^{l}=B^{r} .
\end{array}\right.
$$

or

$$
\left\{\begin{array}{l}
\left(A^{+}+A^{-}\right) X=B \\
\left(\begin{array}{cc}
A^{+} & -A^{-} \\
-A^{-} & A^{+}
\end{array}\right)\left(\begin{array}{l}
X^{l} \\
X^{r}
\end{array}\right)=\left(\begin{array}{l}
B^{l} \\
B^{r}
\end{array}\right) .
\end{array}\right.
$$

Theorem 3.2 Let $S$ belong to $R^{m \times n}$ and $C$ belong to $R^{m \times p}$. Then the minimal $X^{*}$ solution of the matrix equation $S X=C$ is expressed by

$$
X^{*}=S^{\perp} C
$$

where $S^{\perp}$ is the Moore-Penrose generalized inverse[20] of matrix $S$.

Proof. The proof is straight forward.

In order to solve the fuzzy matrix equation (5), we need to consider the systems of linear equations (6). It seems that we have obtained the minimal solution of the function linear system (10) as 


$$
\left\{\begin{array}{l}
X=A^{\perp} B \\
\left(\begin{array}{l}
X^{l} \\
X^{r}
\end{array}\right)=\left(\begin{array}{cc}
A^{+} & -A^{-} \\
-A^{-} & A^{+}
\end{array}\right)^{\perp}\left(\begin{array}{l}
B^{l} \\
B^{r}
\end{array}\right) .
\end{array}\right.
$$

Definition 3.1 Let $\tilde{X}=\left(X, X^{l}, X^{r}\right)$. If $\left(X, X^{l}, X^{r}\right)$ is the minimal solution of Eqs.(10), such that $X^{l} \geq 0, X^{r} \geq 0$, we call $\tilde{X}=\left(X, X^{l}, X^{r}\right)$ is a strong LR fuzzy minimal solution of fuzzy equation (5). Otherwise, the said to a weak LR fuzzy minimal solution of fuzzy matrix equation (5) given by $\quad \tilde{X}=\tilde{x}_{i j}$,

where

$$
\begin{aligned}
\tilde{x}_{i j}= & \left\{\begin{array}{l}
\left(x_{i j}, x^{l}{ }_{i j}, x^{r}{ }_{i j}\right), x^{l}{ }_{i j}>0, x^{r}{ }_{i j}>0, \\
\left(x_{i j}, 0, \max \left\{-x^{l}{ }_{i j}, x^{r}{ }_{i j}\right\}\right), x^{l}{ }_{i j}<0, x^{r}{ }_{i j}>0, \\
\left(x_{i j}, \max \left\{x^{l}{ }_{i j},-x^{r}{ }_{i j}\right\}, 0\right), x^{l}{ }_{i j}>0, x^{r}{ }_{i j}<0, \\
\left(x_{i j},-x^{l}{ }_{i j},-x^{r}{ }_{i j}\right), x^{l}{ }_{i j}<0, x^{r}{ }_{i j}<0 .
\end{array}\right. \\
& i, j=1, \cdots, m .
\end{aligned}
$$

\section{Theorem 3.3 If}

$\left.\left.\left(A^{+}-A^{-}\right)^{+}+\left(A^{+}+A^{-}\right)^{+}\right) \geq 0,\left(A^{+}-A^{-}\right)^{+}-\left(A^{+}+A^{-}\right)^{+}\right) \geq 0$ the fuzzy matrix equation (6) has a strong LR fuzzy minimal solution as follows:

$$
\tilde{X}=\left(X, X^{l}, X^{r}\right)
$$

where

$$
\left\{\begin{array}{l}
X=A^{\perp} B, \\
\left(\begin{array}{l}
X^{l} \\
X^{r}
\end{array}\right)=\left(\begin{array}{cc}
A^{+} & -A^{-} \\
-A^{-} & A^{+}
\end{array}\right)^{\perp}\left(\begin{array}{l}
B^{l} \\
B^{r}
\end{array}\right) .
\end{array}\right.
$$

Proof. Since $B^{l}$ and $B^{r}$ are the left and right spreads fuzzy matrix $\widetilde{B}, B^{l} \geq 0$ and $B^{r} \geq 0$. It means $\left(B^{l}, B^{r}\right)$ is a non negative matrix.

$$
\begin{aligned}
& \text { Let } \\
& S^{+}=\left(\begin{array}{ll}
E & F \\
F & E
\end{array}\right) \\
& =\frac{1}{2}\left(\begin{array}{ll}
\left(A^{+}-A^{-}\right)^{+}+\left(A^{+}+A^{-}\right)^{+} & \left(A^{+}-A^{-}\right)^{+}-\left(A^{+}+A^{-}\right)^{+} \\
\left(A^{+}-A^{-}\right)^{+}-\left(A^{+}+A^{-}\right)^{+} & \left(A^{+}-A^{-}\right)^{+}+\left(A^{+}+A^{-}\right)^{+}
\end{array}\right) .
\end{aligned}
$$

We know the condition that $S^{\perp} \geq 0$ is equivalent to $E \geq 0$ and $F \geq 0$.

The product of two non negative matrices

$$
\begin{aligned}
& \left(\begin{array}{l}
X^{l} \\
X^{r}
\end{array}\right)=\left(\begin{array}{cc}
A^{+} & -A^{-} \\
-A^{-} & A^{+}
\end{array}\right)^{\perp}\left(\begin{array}{l}
B^{l} \\
B^{r}
\end{array}\right)=\frac{1}{2}\left(\begin{array}{cc}
E & F \\
F & E
\end{array}\right)\left(\begin{array}{l}
B^{l} \\
B^{r}
\end{array}\right) \\
& =\frac{1}{2}\left(\begin{array}{l}
E B^{l}+F B^{r} \\
F B^{l}+E B^{r}
\end{array}\right) \geq O
\end{aligned}
$$

is non negative in nature. i.e. $X^{l} \geq 0$ and $X^{r} \geq 0$.

\section{NUMERICAL EXAMPLES}

Example 4.1. Consider the following fuzzy matrix system

$$
\left(\begin{array}{ll}
\tilde{x}_{11} & \tilde{x}_{12} \\
\tilde{x}_{21} & \tilde{x}_{22}
\end{array}\right)\left(\begin{array}{cc}
1 & -1 \\
-1 & 1
\end{array}\right)=\left(\begin{array}{ll}
(3,1,1) & (2,1,2) \\
(2,1,1) & (1,0,1)
\end{array}\right)
$$

Let

$$
\begin{gathered}
\tilde{X}=\left(X, X^{l}, X^{r}\right) \\
=\left(\left(\begin{array}{ll}
x_{11} & x_{12} \\
X_{21} & x_{22}
\end{array}\right),\left(\begin{array}{ll}
x^{l}{ }_{11} & x^{l}{ }_{12} \\
x^{l}{ }_{21} & x^{l}{ }_{22}
\end{array}\right),\left(\begin{array}{ll}
x^{r}{ }_{11} & x^{r}{ }_{12} \\
x^{r}{ }_{21} & x^{r}{ }_{22}
\end{array}\right)\right), \\
A=A^{+}+A^{-}=\left(\begin{array}{ll}
1 & 0 \\
0 & 1
\end{array}\right)+\left(\begin{array}{cc}
0 & -1 \\
-1 & 0
\end{array}\right) .
\end{gathered}
$$

and

$$
\widetilde{B}=\left(B, B^{l}, B^{r}\right)=\left(\left(\begin{array}{ll}
3 & 2 \\
2 & 3
\end{array}\right),\left(\begin{array}{ll}
1 & 1 \\
1 & 0
\end{array}\right),\left(\begin{array}{ll}
1 & 2 \\
1 & 1
\end{array}\right)\right) .
$$

By the Theorem 3.1., the original fuzzy matrix equation is equivalent to the following linear system

$$
\left\{\begin{array}{l}
\left(A^{+}+A^{-}\right) X=B, \\
\left(\begin{array}{cc}
A^{+} & -A^{-} \\
-A^{-} & A^{+}
\end{array}\right)\left(\begin{array}{l}
X^{l} \\
X^{r}
\end{array}\right)=\left(\begin{array}{l}
B^{l} \\
B^{r}
\end{array}\right)
\end{array}\right.
$$

The matrix $S$ is obviously not invertible one, its M-P inverse is

$$
S^{+}=\frac{1}{4}\left(\begin{array}{llll}
1 & 0 & 0 & 1 \\
0 & 1 & 1 & 0 \\
0 & 1 & 1 & 0 \\
1 & 0 & 0 & 1
\end{array}\right) \geq 0
$$

From Eqs. (10), the minimal solution of model is as follows:

$$
X=A^{\perp} B=\left(\begin{array}{ll}
0.25 & -0.25 \\
0.25 & -0.25
\end{array}\right)\left(\begin{array}{ll}
3 & 2 \\
2 & 3
\end{array}\right)=\left(\begin{array}{ll}
0.25 & -0.25 \\
0.25 & -0.25
\end{array}\right),
$$




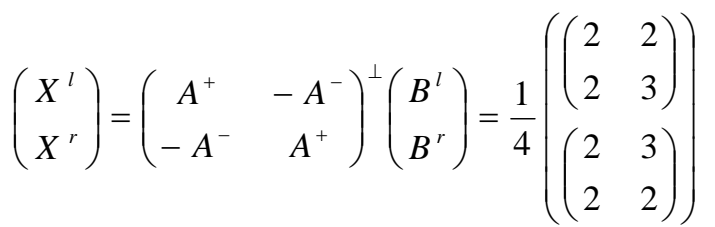

i.e.,

$$
\left(\begin{array}{ll}
\tilde{x}_{11} & \tilde{x}_{12} \\
\tilde{x}_{21} & \tilde{x}_{22}
\end{array}\right)=\left(\begin{array}{ll}
(0.25,0.50,0.50) & (-0.25,0.50,0.75) \\
(0.25,0.50,0.50) & (-0.25,0.75,0.50)
\end{array}\right)
$$

Since $\tilde{X}$ is an appropriate LR fuzzy numbers matrix, we obtained the solution of the fuzzy matrix system is a strong LR fuzzy solution by Definition 8 .

\section{CONCLUSION}

In this work, we discussed a class of LR fuzzy matrix equations $A \tilde{X}=\tilde{B}$ by a full matrix method. The model we constructed for solving original fuzzy matrix system was made of two crisp systems of linear matrix equations. The existence condition of strong LR fuzzy solution was also studied. Our result will enrich fuzzy linear system theory and can be applied to solve all kinds of fuzzy matrix equations.

\section{ACKNOWLEDGEMENT}

This research work was supported by the National Natural Science Foundation of China (no.11761062) and the Scientific Research Project of Gansu Province Colleges and Universities (no.2017A-058).

\section{REFERENCES}

[1] L.A. Zadeh, "The concept of a linguistic variable and its application to approximate reasoning”, Information Science, Vol 8, pp. 199-249, 1975.

[2] D. Dubois, H. Prade, “Operations on fuzzy numbers”, Journal of Systems Science, Vol 9, pp. 613-626,1978.

[3] S. Nahmias, Fuzzy variables, "Fuzzy Sets and Systems," Vol 2, pp. 97-111, 1978.

[4] M.L. Puri, D.A. Ralescu, "Differentials for fuzzy functions”, Journal of Mathematics Analyisis and Application, Vol 91 , pp. 552-558, 1983.

[5] R. Goetschel, W. Voxman, "Elementary calculus", Fuzzy Sets and Systems, Vol 18, pp. 31-43, 1986.

[6] C.X. Wu, M. Ma, "Embedding problem of fuzzy number space: Part I", Fuzzy Sets and Systems, Vol 44, pp. 33-38, 1991.

[7] C.X. Wu, M. Ma, "Embedding problem of fuzzy number space: Part III", Fuzzy Sets and Systems, Vol 46, pp. 281-286, 1992.

[8] M. Friedman, M. Ma, A. Kandel, "Fuzzy linear systems”, Fuzzy Sets and Systems, Vol 96, pp. 201-209, 1998.

[9] S. Abbasbandy, M. Otadi, M.Mosleh, "Minimal solution of general dual full linear systems,Chaos”, Solitions and Fractals, Vol 29, pp. 638-652, 2008.

[10] T. Allahviranloo, F. H. Lotfi, M. K. Kiasari,M. Khezerloo, "On the fuzzy solution of LR fuzzy linear systems”, Applied Mathematical and Modelling, Vol 37, pp. 1170-1176, 2013.

[11] R. Nuraei, T. Allahviranloo, M. Ghanbari, "Finding an inner estimation of the soluion set of a fuzzy linear systems”, Applied Mathematical and Modelling, Vol 37, pp. 165-177, 2009.

[12] B. Zheng, K. Wang, “General fuzzy linear systems”, Appl. Math. Comput., Vol 181, pp. 1276-1286, 2006.
[13] N. Babbar, A. Kumar, A. Bansal, "Solving full fuzzy linear systems with arbitrary triangular fuzzy numbers”, Soft Computing, Vol 17, pp. 691-702, 2013.

[14] R. Ghanbari, "Solutions of fuzzy LR algeraic linear systems using linear programs", Applied Mathematical and Modelling, Vol 39, pp. 5164-5173, 2015.

[15] T. Allahviranloo, N. Mikaeilvand, M. Barkhordary, "Fuzzy linear matrix equations", Fuzzy Optimization and Decision Making,Vol 37, pp. 165-177, 2009.

[16] Z.T. Gong, X.B. Guo, "Inconsistent fuzzy matrix equations and its fuzzy least squares solutions,” Applied Mathematical Modelling, Vol.35, pp. 1456-1469, 2011.

[17] M. Otadi, M.Mosleh, "Solving fuzzy matrix equations", Applied Mathematical Modelling, Vol. 36, pp. 6114-6121, 2012.

[18] X.B. Guo, K. Zhang, "Minimal solution of complex fuzzy linear systems”, Advances in Fuzzy Systems, pp. 1-9, 2016.

[19] X.B. Guo, K. Zhang, "Solving fuzzy matrix equation of the form XA=B”, Journal of Intelligent and Fuzzy Systems, Vol. 32, pp. 2771-2778, 2017.

[20] Ben-Israel, T.N.E. Greville, “Generalized inverses: Theory and applications, second edition”, Springer-Verlag, 2003. 